\title{
Oxidative stress and protective mechanisms in erythrocytes in relation to Plasmodium vinckei load
}

\author{
(malaria/reactive oxygen species/parasite density/gradient separation)
}

\author{
Roland Stocker* ${ }^{*}$, Nicholas H. Hunt ${ }^{\dagger}$, Gary D. Buffinton ${ }^{\dagger}$, Maurice J. Weidemann*, \\ Peter H. Lewis-Hughes*, and Ian A. Clark* \\ *Departments of Biochemistry and Zoology, Faculty of Science and †Experimental Pathology, John Curtin School of Medical Research, \\ Australian National University, Canberra, ACT 2601, Australia \\ Communicated by Frank Fenner, August 8, 1984
}

\begin{abstract}
The protection of mouse erythrocytes (RBC) parasitized with Plasmodium vinckei vinckei against activated oxygen species was examined in relation to the intraerythrocytic parasite load. RBC from highly infected animals were separated by density gradient centrifugation into six bands with increasing parasite content and with parasitemias ranging from $17 \%$ to $100 \%$. Increase in parasite load was accompanied by a decrease in the activities of the enzymes superoxide dismutase (EC 1.15.1.1), catalase (EC 1.11.1.6), glutathione peroxidase (EC 1.11.1.9), glutathione reductase [NAD(P)H] (EC 1.6.4.2), and NADH-methemoglobin reductase (EC 1.6.2.2; NADH:ferricytochrome $b_{5}$ oxidoreductase) in the RBC lysates. In contrast, the total amount of reduced glutathione increased in the highly parasitized bands. Furthermore, the vitamin $E$ content of all RBC bands, including the one that contained mainly nonparasitized erythrocytes, was 3- to 5-fold higher than that of control noninfected RBC. Increasing parasite load was accompanied by an increase in the production of malonyldialdehyde, indicating enhanced lipid peroxidation. Our results indicate that oxidative stress is experienced by all $\mathbf{R B C}$ during a malarial infection and is accompanied by a variety of changes in the antioxidant defense mechanisms of the host and the parasite. Furthermore, it appears that the plasma membrane of the host cell is better protected against oxidative injury than are the membranes surrounding the parasite.
\end{abstract}

Malarial infection is accompanied by a variety of biological responses on the part of the host, including the activation of its cellular immune system. Experiments with Plasmodium falciparum and Plasmodium berghei in vitro suggest that phagocytic cells from spleen (1), peritoneal cavity $(1,2)$, and peripheral blood (3-6) ingest parasitized erythrocytes (PRBC) or free merozoites as part of their role in cell-mediated immunity. Phagocytosis by macrophages and polymorphonuclear leukocytes is accompanied by a respiratory burst, which gives rise to the production of reactive oxygen species (ROS) $(7,8)$ that may be involved in the processes that lead to hemolysis and intraerythrocytic death of malarial parasites (9-11). Experiments with the murine parasite Plasmodium yoelii in vitro have shown that lymphokine-treated macrophages release hydrogen peroxide extracellularly upon phagocytosis of PRBC (12). This and other ROS could affect host tissues, including unparasitized $R B C$ as well as PRBC and their contents. PRBC not only trigger the oxidative burst of macrophages but also act as target cells in a cytotoxic assay, resulting in intraerythrocytic death of the parasite (12). There is some evidence that the host RBC is exposed to oxidative stress during malarial infection (13), and it has been argued that decreased protection against oxidative damage may inhibit parasite growth in thalassemic

The publication costs of this article were defrayed in part by page charge payment. This article must therefore be hereby marked "advertisement" in accordance with 18 U.S.C. $\$ 1734$ solely to indicate this fact.
RBC (14) and in erythrocytes deficient in glucose-6-phosphate dehydrogenase $(15,16)$ or vitamin E $(15)$. Experiments designed to obtain information about $\mathrm{RBC}$ susceptibility to oxidative stress during malarial infection have concentrated mainly on the stability and oxidation/reduction status of glutathione (GSH = reduced form; GSSG = oxidized form) in PRBC $(16,17)$, but the effect of parasitemia upon the full range of antioxidant systems has yet to be explored.

In this study RBC from the blood of highly parasitized mice were separated on the basis of parasite load and examined for their antioxidant activities. Our results are consistent with the exposure of unparasitized RBC to oxidative stress and show that, with increased parasite content, complex changes occur in the antioxidant defense mechanisms within the PRBC.

\section{METHODS}

Male CBA/CaH mice, 6-10 weeks old, were infected by i.p. injection of $10^{6}$ Plasmodium vinckei subsp. vinckei PRBC (9).

Blood from control or infected mice, the latter showing 70-90\% parasitemia, was collected into sodium heparin $(20$ units $/ \mathrm{ml}$ of blood). RBC from infected mice were separated as described by others (18). Briefly, 1-2 $\mathrm{ml}$ of infected blood was layered onto discontinuous gradients of Dextran T 40 (Pharmacia) in phosphate-buffered saline $/ 5 \mathrm{mM}$ glucose, $\mathrm{pH}$ 7.2 , containing $4 \mathrm{ml}$ of $40 \%$ Dextran T $40,7 \mathrm{ml}$ of $28 \%, 6 \mathrm{ml}$ of $23 \%, 5 \mathrm{ml}$ of $19 \%, 5 \mathrm{ml}$ of $17 \%$, and $4 \mathrm{ml}$ of $10 \%$. The gradients were centrifuged for $45 \mathrm{~min}\left(15,000 \times g\right.$ at $\left.4^{\circ} \mathrm{C}\right)$ in an SW 27 rotor, which separated the blood into six different bands with the most highly parasite-loaded RBC on top (hereafter referred to as band 1) and the mainly nonparasitized RBC (band 6) on the bottom. Centrifugation of control blood (from uninfected mice) resulted in only one distinct band, corresponding to band 6 of the infected blood. Cells were recovered and washed twice with phosphate-buffered saline $/ 5 \mathrm{mM}$ glucose before their concentration was adjusted for further assays. To characterize the cellular constituents of the different bands, smears were prepared with the aid of a Cytospin cell centrifuge (Shandon Southern, Astmoor, England) and stained with Harleco's Diff-Quik stain set (American Hospital Supplies, Sydney). Parasite and reticulocyte numbers were assessed by counting 300-400 cells.

Enzyme activities of the different washed bands were assayed by published methods: superoxide dismutase (SOD; EC 1.15.1.1) (19); catalase (EC 1.11.1.6) (20); and NADHmethemoglobin reductase (EC 1.6.2.2; NADH:ferricytochrome $b_{5}$ oxidoreductase), glutathione peroxidase (EC

Abbreviations: GSH and GSSG, reduced and oxidized forms of glutathione, respectively; RBC, erythrocyte(s); PRBC, parasitized erythrocyte(s); ROS, reactive oxygen species; SOD, superoxide dismutase; $\mathrm{tBuO}_{2} \mathrm{H}, t$-butyl hydroperoxide.

¥To whom reprint requests should be addressed. 
Table 1. Characterization of RBC bands, after density centrifugation of blood from highly infected animals over a discontinuous Dextran T $\mathbf{4 0}$ gradient

\begin{tabular}{|c|c|c|c|c|c|c|c|}
\hline \multirow[b]{2}{*}{ Band } & \multirow[b]{2}{*}{ Parasitemia* } & \multirow[b]{2}{*}{ Reticulocytes* } & \multirow[b]{2}{*}{ Leucocytes* } & \multicolumn{4}{|c|}{ Number of parasites/100 RBC ${ }^{\dagger}$} \\
\hline & & & & Ring & Trophozoite & Schizont & Total \\
\hline 6 & $17.3 \pm 4.8$ & $11 \pm 6$ & $0-1$ & $\begin{array}{c}13.8 \pm 3.1 \\
(73.4)\end{array}$ & $\begin{array}{l}5.0 \pm 3.5 \\
(26.6)\end{array}$ & 0 & $18.8 \pm 6.2$ \\
\hline 5 & $71.0 \pm 26.3$ & $16 \pm 6$ & $0-1$ & $\begin{array}{c}7.9 \pm 3.2 \\
(9.0)\end{array}$ & $\begin{array}{l}78.1 \pm 27.2 \\
(88.2)\end{array}$ & $\begin{array}{c}2.0 \pm 1.2 \\
(2.3)\end{array}$ & $88.1 \pm 25.1$ \\
\hline 4 & $96.2 \pm 13.6$ & $5 \pm 2$ & $0-1$ & $\begin{array}{c}3.9 \pm 3.6 \\
(2.3)\end{array}$ & $\begin{array}{c}162.1 \pm 23.5 \\
(91.7)\end{array}$ & $\begin{array}{c}10.4 \pm 4.32 \\
(6.0)\end{array}$ & $176.3 \pm 23.7$ \\
\hline 3 & $99.6 \pm 6.3$ & $3 \pm 1$ & $0-1$ & $\begin{array}{c}1.2 \pm 1.2 \\
(0.5)\end{array}$ & $\begin{array}{c}236.7 \pm 29.7 \\
(93.5)\end{array}$ & $\begin{array}{c}15.2 \pm 5.8 \\
(6.0)\end{array}$ & $253.1 \pm 33.2$ \\
\hline 2 & $99.4 \pm 3.7$ & $1 \pm 1$ & 0 & $\begin{array}{c}5.3 \pm 6.1 \\
(1.7)\end{array}$ & $\begin{array}{c}280.9 \pm 12.8 \\
(90.7)\end{array}$ & $\begin{array}{c}23.3 \pm 12.8 \\
(7.6)\end{array}$ & $309.6 \pm 39.9$ \\
\hline 1 & $99.0 \pm 11.0$ & $1 \pm 1$ & 0 & $\begin{array}{c}1.8 \pm 2.3 \\
(0.5)\end{array}$ & $\begin{array}{c}351.9 \pm 31.6 \\
(93.2)\end{array}$ & $\begin{array}{c}23.9 \pm 11.0 \\
(6.3)\end{array}$ & $377.6 \pm 33.6$ \\
\hline
\end{tabular}

*Results are expressed as percentages of mean values \pm SD of the total cell content of four different separations.

†Numbers represent mean values \pm SD with percentages of the total numbers of parasites in each band in parenthesis.

1.11.1.9), and glutathione reductase (EC 1.6.4.2) (21). Total hemoglobin and GSH concentration of the freeze-thawed hemolysates of the different bands were measured as described (21). Vitamin E was determined by a fluorimetric method (22).

Malonyldialdehyde was determined as described (23) with slight modification (24).

\section{RESULTS}

The aim of this study was to determine the susceptibility of PRBC to oxidative injury in relation to the intraerythrocytic growth and development of the parasite using density gradient separation of RBC (18) into six different bands (Table 1), each containing RBC with different concentrations of parasites. The RBC of band 6 were mainly nonparasitized $(83 \%)$ or contained parasites at the early stages of development, and band 1 contained the most heavily parasitized RBC (Table 1). Parasites in RBC from all bands (except band 6) were mainly at the trophozoite stage of development, although the absolute numbers of schizonts increased from band 6 to band 1 , and the numbers of ring forms generally decreased. However, the bands did not represent an orderly maturation process, since multiple infections were common in the lessdense bands. Leucocytes were never observed in bands 1 and 2 , and the leucocyte contamination in the remaining bands was always $<1 \%$.

To find out whether increased parasite load, represented by the different bands, is accompanied by an increase in the oxidative stress exerted upon the host cell, we tested in an in vitro system the spontaneous and $t$-butyl hydroperoxide $\left(\mathrm{tBuO}_{2} \mathrm{H}\right)$-induced production of malonyldialdehyde, using this as an index of lipid peroxidation. Incubation of PRBC in phosphate-buffered saline for $2 \mathrm{hr}$ at $37^{\circ} \mathrm{C}$ resulted in spontaneous malonyldialdehyde production (Fig. $1 a$ ). The presence of $1 \mathrm{mM} \mathrm{tBuO} 2 \mathrm{H}$ increased the amount of malonyldialdehyde formed, the greatest effect being observed in band 1 (Fig. $1 b$ ). Both the spontaneous and $\mathrm{tBuO}_{2} \mathrm{H}$-induced production of malonyldialdehyde by all bands tested were inhibited by the iron-chelating drug desferrioxamine (Fig. 1). To test whether increasing parasite load influenced the antioxidant defense mechanisms of the host, we examined the activities of enzymes involved in ROS degradation and measured the concentrations of nonenzymatic antioxidants within the different bands. The activities of SOD, catalase, and GSH peroxidase on a per cell basis decreased progressively as the parasite load increased (Table 2). The activity of GSSG reductase was elevated in the mainly nonparasitized band 6 compared with its activity in control $R B C$, but it decreased with increasing parasite concentration (Table 2 ). In contrast to these changes in enzyme activities, the total content of GSH in all bands was elevated compared with its control value (Table 3). In addition to its cytosolic defense mechanisms, the RBC contains antioxidants within the cell membrane, the most important of these being vitamin $E(25)$. RBC from mice sick with $P$. vinckei infection contained 3-5 times more vitamin $\mathrm{E}$ than did control RBC, irrespective of the parasite load or the proportion of $R B C$ that were parasitized. Band 6 (containing mainly nonparasitized RBC) showed the highest content of vitamin E (Table 3).

\section{DISCUSSION}

ROS are hypothesized to cause certain malarial pathology $(9,26)$ and the intraerythrocytic death of parasites $(9-13)$. These toxic forms of oxygen also are thought to inhibit parasite growth indirectly in some host RBC variants in which antioxidant protection is insufficient (14-16). Evidence that uncompensated oxidative stress is exerted upon PRBC comes from a report showing that methemoglobin and sulfhemoglobin accumulates during $P$. berghei infection, possibly as a result of $\mathrm{H}_{2} \mathrm{O}_{2}$ production by the parasite (13). However, the changes reported were small, and some of the evidence was indirect. Furthermore, other studies $(17,18)$ have

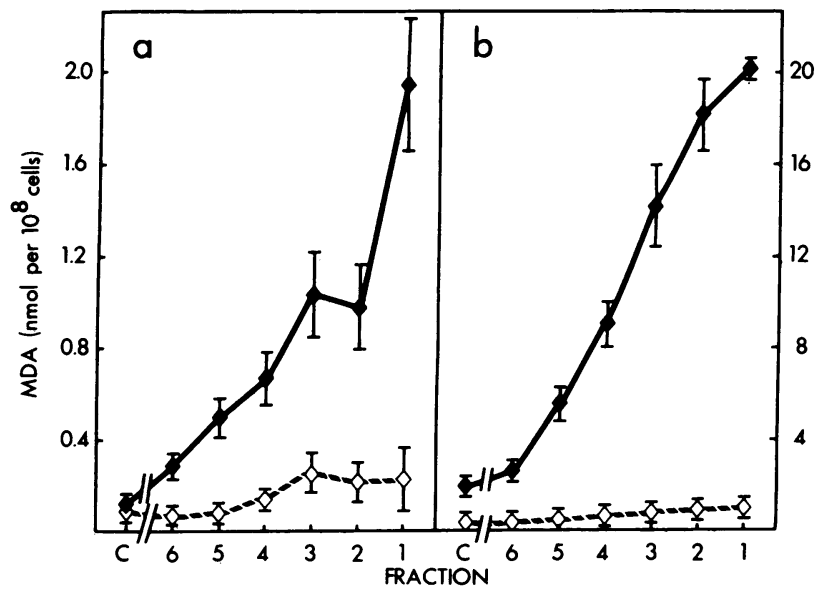

FIG. 1. Production of malonyldialdehyde (MDA) during different stages of intraerythrocytic development of the malarial parasite. (a) Spontaneous MDA production after $2 \mathrm{hr}(\bullet)$. (b) $\mathrm{tBuO}_{2} \mathrm{H}(1 \mathrm{mM})$ induced MDA production after $2 \mathrm{hr}(\bullet)$. Inhibition by a $30-\mathrm{min}$ preincubation with desferrioxamine $(4 \mathrm{mM})$ before addition of either phosphate-buffered saline $(a)$ or $1 \mathrm{mM} \mathrm{tBuO} \mathrm{H}_{2} \mathrm{H}(\diamond)$. C, control RBC. Fractions 1-6 were separated, characterized and assayed as described. Data represent means $\pm \operatorname{SEM}(n=5)$. 
Table 2. Antioxidant enzyme activities in PRBC in relation to parasite load

\begin{tabular}{cccccc}
\hline Band & SOD $^{*}$ & Catalase* $^{*}$ & $\begin{array}{c}\text { MADH- } \\
\text { RetHb- }\end{array}$ & $\begin{array}{c}\text { GSH } \\
\text { peroxidase* }\end{array}$ & $\begin{array}{c}\text { GSSG } \\
\text { reductase* }\end{array}$ \\
\hline 6 & $82 \pm 17$ & $102 \pm 21$ & $98 \pm 22$ & $85 \pm 41$ & $178 \pm 38^{\ddagger}$ \\
& $(5)$ & $(3)$ & $(5)$ & $(3)$ & $(3)$ \\
5 & $81 \pm 19$ & $104 \pm 17$ & $104 \pm 19$ & $108 \pm 20$ & $130 \pm 49$ \\
& $(5)$ & $(3)$ & $(5)$ & $(3)$ & $(3)$ \\
4 & $78 \pm 23$ & $95 \pm 16$ & $74 \pm 16^{\dagger}$ & $82 \pm 17$ & $109 \pm 49$ \\
& $(4)$ & $(3)$ & $(5)$ & $(3)$ & $(3)$ \\
3 & $79 \pm 14^{\dagger}$ & $77 \pm 30$ & $72 \pm 17^{\dagger}$ & $69 \pm 16$ & $86 \pm 39$ \\
& $(5)$ & $(3)$ & $(5)$ & $(3)$ & $(3)$ \\
2 & $72 \pm 10^{\dagger}$ & $68 \pm 18$ & $56 \pm 15^{\dagger}$ & $41 \pm 13$ & $65 \pm 27^{\dagger}$ \\
& $(5)$ & $(3)$ & $(5)$ & $(3)$ & $(3)$ \\
1 & $69 \pm 16^{\dagger}$ & $56 \pm 10^{\dagger}$ & $45 \pm 19^{\dagger}$ & $28 \pm 44^{\dagger}$ & $59 \pm 20^{\dagger}$ \\
& $(4)$ & $(3)$ & $(5)$ & $(3)$ & $(3)$ \\
\hline
\end{tabular}

*Enzyme activities are expressed as the mean $\pm \mathrm{SD}(n$ in parentheses) of the percentages of the relevant control values (RBC from uninfected animals). The values for control (100\%) activity per $10^{9}$ cells are: SOD, 63.4 units; catalase, 552 units; NADH-methemoglobin reductase (NADH-MetHb-red), $0.061 \mathrm{~mol}$ of $\mathrm{Hb}$ reduced/ min; GSH peroxidase, $0.76 \mathrm{~mol}$ of NADPH oxidized/min; GSSG reductase, $0.17 \mathrm{~mol}$ of NADPH oxidized $/ \mathrm{min}$.

†Value is significantly smaller than the control value $(0.0005<P<$ 0.05 ).

¥Value is significantly higher than the control value $(P<0.0125)$.

shown that GSH stability in PRBC was enhanced, thus throwing into doubt the occurrence of oxidative stress in murine and human malaria. These authors expressed GSH levels and enzyme activities in terms of protein (18) or hemoglobin (17) concentration. Calculation on this basis can result in misinterpretation, since these two parameters also change during the course of malarial infection (Table 3 ; rêf. 18). Therefore, we examined the potential of PRBC in each band to degrade ROS and the susceptibility of their membrane lipids to oxidation and expressed the results on a per cell basis. Since the results were derived from a cellular system composed of two major components (i.e., the parasite and the "residual" host cell), it is difficult to attribute any particular observation to one or other of these "compartments."

The steady decrease in the activities of SOD, catalase, and GSH peroxidase within PRBC as the parasite burden increased (Table 2) together with the failure of the enzyme levels to match the 4-fold increase in parasitemia from band 6 to

Table 3. Antioxidants within PRBC in relation to parasite load

\begin{tabular}{cccc}
\hline Band & Hemoglobin* & GSH* $^{*}$ & Vitamin E* \\
\hline 6 & $84 \pm 12$ & $151 \pm 13^{\ddagger}$ & $544 \pm 211^{\ddagger}$ \\
& $(5)$ & $(3)$ & $(6)$ \\
5 & $89 \pm 16$ & $157 \pm 28$ & $379 \pm 194^{\ddagger}$ \\
& $(6)$ & $(3)$ & $(6)$ \\
4 & $66 \pm 16^{\dagger}$ & $205 \pm 26^{\ddagger}$ & $330 \pm 130^{\ddagger}$ \\
& $(6)$ & $(3)$ & $(6)$ \\
3 & $44 \pm 17^{\dagger}$ & $212 \pm 4^{\ddagger}$ & $394 \pm 252^{\ddagger}$ \\
& $(6)$ & $(3)$ & $(6)$ \\
2 & $32 \pm 15^{\dagger}$ & $231 \pm 57^{\ddagger}$ & $377 \pm 167^{\ddagger}$ \\
& $(6)$ & $(3)$ & $(6)$ \\
1 & $22 \pm 9^{\dagger}$ & $174 \pm 9 \ddagger$ & $470 \pm 179^{\ddagger}$ \\
& $(5)$ & $(3)$ & $(6)$
\end{tabular}

*Values represent arithmetic mean $\pm \mathrm{SD}$ ( $n$ in parentheses), expressed as the percentage of control (RBC from uninfected animals). The corresponding values for control (100\%) values per $10^{9}$ cells are: hemoglobin, $20.3 \mathrm{mg}$; GSH, $117 \mathrm{nmol}$; and vitamin $\mathrm{E}$ $0.17 \mu \mathrm{g}$.

tValue is significantly smaller than control value $(0.0005<P<$ 0.01 ).

$\ddagger$ Value is significantly higher than control value $(0.0005<P<0.05)$. band 5 , indicate that these enzymes are probably of erythrocytic origin, since their activity losses approximately paralleled the overall loss in total protein concentration (18). This observation implies that the parasite digests RBC cytosolic enzymes as well as the hemoglobin it has pinocytosed. The high activity of GSSG reductase in the mainly nonparasitized band 6 suggests that any contribution of parasite GSSG reductase to the overall activity is likely to be of minor importance. Despite this decrease in the activity of GSSG reductase, the GSH content increased from band 6 to band 2, showing elevated levels in all bands from infected blood compared with control blood (Table 3). Therefore, it seems likely that a significant portion of the GSH is associated with the parasite itself. It is possible that, in conjunction with the activity of parasite GSSG reductase, the presence of malarial NADP-specific glutamate dehydrogenase (EC 1.4.1.3) (27) together with increased activity of $\gamma$-glutamylcysteine synthetase (EC 6.3.2.2) (18) might be responsible for the increased GSH level.

The possibility that the cytosolic compartment of the PRBC is under oxidative stress remains open. Several authors $(28,29)$ have argued that the hemoglobin present within intact $\mathrm{RBC}$ is protected against oxidation by $\mathrm{H}_{2} \mathrm{O}_{2}$ as long as GSH is available as an electron donor for GSH peroxidase. When GSH is oxidized faster than it can be regenerated, catalase and hemoglobin compete for the excess $\mathrm{H}_{2} \mathrm{O}_{2}$ and formation of methemoglobin can occur. Such an explanation is not ruled out by the present work, since the results indicate that the major portion of the GSH is located within the parasite, although we cannot exclude the possibility that some GSH belongs to the "residual" RBC. We have been unable to detect accumulated methemoglobin in any of the bands (data not shown), although such accumulation has been reported in Plasmodium berghei-PRBC (13). Enhanced recycling of methemoglobin to oxyhemoglobin, through increased activity of NADH-methemoglobin reductase, is unlikely to be responsible for the lack of methemoglobin accumulation in this case, since the activity of this enzyme actually decreased from band 6 to band 1 (Table 2). These considerations imply that the cytosolic compartment of the PRBC is not under oxidative stress under the experimental conditions used here.

Incubation of PRBC in vitro resulted in the spontaneous production of malonyldialdehyde, the amount detected being positively correlated with parasite load (Fig. 1a). Thus, increased numbers of parasites within $\mathrm{RBC}$ are accompanied by increased lipid peroxiation. A striking observation was the difference in content of vitamin $\mathrm{E}$, the most important lipid-soluble antioxidant (25). The vitamin E content of RBC from sick mice increased to 3 - to 5-fold above normal levels, irrespective of the degree of parasitemia (Table 3). Band 6, in which only $17 \%$ of the RBC were infected, mostly with young parasites, contained at least as much vitamin $\mathrm{E}$ as bands with a higher parasite content. Thus, the vitamin $E$ content of the different bands is not correlated directly with parasite load but may well be a protective response to a hostinduced oxidative stress. From these results we conclude that the plasma membrane of PRBC is reasonably well protected against oxidative injury compared with the membranes surrounding the parasite and, thus, is unlikely to be the origin of the increase in spontaneous malonyldialdehyde production.

There is evidence that inhalation of nitrogen dioxide, which initiates free radical reactions and causes oxidant damage to lungs, mobilizes vitamin $\mathrm{E}$ from fat stores to the lung (30). This has been suggested to be a protective mechanism (31), and it would serve the same purpose as the antioxidant enzymes induced in lung tissue after chronic exposure to high concentrations of oxygen (32). The increased vitamin $\mathrm{E}$ content in parasitized or unparasitized $\mathrm{RBC}$ from mice ill 
with malaria may be a similar response and is consistent with our earlier suggestion (9) that host tissues may come under free radical-induced oxidative attack from leucocytes as the disease progresses. This interpretation is strengthened by our recent experiments (unpublished data) showing that the exposure of normal mice to divicine, a source of free radicalinduced oxidative stress (24), increases vitamin $E$ in their plasma and RBC.

As the parasite load increases within the $\mathrm{RBC}$, up to $80 \%$ of the total hemoglobin is digested (Table 3). The first step in the process of hemoglobin degradation, which takes place within the food vacuoles of the parasite, includes the oxidation of oxyhemoglobin to methemoglobin (33) with consequent formation of ROS. Thus, the high oxidative capacity of the food vacuoles might contribute to the spontaneous malonyldialdehyde production observed in PRBC. Whatever the source of malonyldialdehyde production might be, the process appears to be iron-dependent, since the chelating agent desferrioxamine inhibits both the spontaneous and $\mathrm{tBuO}_{2} \mathrm{H}$-induced lipid peroxidation (Fig. 1). Generation of oxygen-centered radicals through a process catalyzed by transition metals has been well documented (34).

These studies have focused on the susceptibility of PRBC toward oxidative injury in relation to parasite load and development. Our results suggest that, during the course of malarial infection, all RBC are exposed to oxidative stress. They respond by attempting to protect themselves against the damage caused by ROS. Furthermore, the membranes surrounding the parasite might contribute greatly to the increased lipid peroxidation observed during parasite development in the presence and absence of exogenous ROS. This would explain why, during the natural course of an infection or after administration of chemicals that generate oxygenderived free radicals, parasites with damaged membranes are observed inside host RBCs that retain intact plasma membranes $(24,35)$.

During the preparation of this manuscript we became aware of an abstract (36) reporting increased levels of vitamin $\mathrm{E}$ in human and murine malaria-infected RBC.

We thank Mrs. K. Rabl and Mrs. D. Kjeldsen for secretarial assistance. This study was supported by the malaria component of the United Nations Development Programme/World Bank/World Health Organisation's Special Programme for Research and Training in Tropical Diseases and by the National Health and Medical Research Council of Australia. R.S. and G.D.B. were recipients of Australian National University and Commonwealth Postgraduate Awards, respectively.

1. Shear, H. L., Nussenzweig, R. S. \& Bianco, C. (1979) J. Exp. Med. 149, 1288-1289.

2. Makimura, S. \& Suzuki, N. (1977) Res. Bull. Obihiro Univ. 10, 401-406.

3. Trubowitz, S. \& Masek, B. (1968) Science 162, 273-274.

4. Celado, A., Cruchaud, A. \& Perrin, L. H. (1983) J. Parasitol. 69, 49-53.

5. Vernes, A. (1980) Lancet ii, 1297-1298.
6. Khusmith, S., Druilhe, P. \& Gentilini, M. (1982) Infect. Immun. 35, 874-879.

7. Babior, B. M. (1978) New Engl. J. Med. 298, 659-668.

8. Badway, J. A. \& Karnovsky, M. L. (1980) Annu. Rev. Biochem. 49, 695-726.

9. Clark, I. A. \& Hunt, N. H. (1983) Infect. Immun. 39, 1-6.

10. Clark, I. A., Cowden, W. B. \& Butcher, G. A. (1983) Lancet i, 234.

11. Dockrell, H. M. \& Playfair, J. H. (1983) Infect. Immun. 39, 456-459.

12. Ockenhouse, C. F. \& Shear, H. L. (1984) J. Immunol. 132 424-431.

13. Eaton, J. W. \& Eckman, J. R. (1979) in Biochemical and Clinical Aspects of Oxygen, ed. Caughey, W. (Academic, New York), pp. 825-837.

14. Friedman, M. J. (1979) Nature (London) 280, 245-247.

15. Eaton, J. W., Eckman, J. R., Berger, E. \& Jacob, H. S. (1976) Nature (London) 264, 758-760.

16. Eckman, J. R. \& Eaton, J. W. (1979) Nature (London) 278 , 754-756.

17. Roth, E. F., Jr., Raventos-Suarez, C., Perkins, M. \& Nagel, R. L. (1982) Biochem. Biophys. Res. Commun. 109, 355-362.

18. Picard-Maureau, A., Hempelmann, E., Krammer, G., Jakisch, R. \& Jung, A. (1975) Tropenmed. Parasitol. 26, 405-416.

19. Crapo, J. D., McCord, J. M. \& Fridovich, I. (1978) in Methods in Enzymology, eds. Fleischer, S. \& Packer, L. (Academic, New York), Vol. 53, pp. 382-393.

20. Aebi, H. (1974) in Methods of Enzymatic Analysis, ed. Bergmeyer, H. V. (Verlag Chemie, Weinheim, F.R.G.), pp. 673683.

21. Beutler, E. (1971) in A Manual of Biochemical Methods (Grune \& Stratton, New York), pp. 13-14; 103-105.

22. Taylor, S. L., Lamden, M. P. \& Tappel, A. L. (1976) Lipids 11, 530-538.

23. Stocks, J., Offerman, E. L., Modell, C. B. \& Dormandy, T. L. (1972) Br. J. Haematol. 23, 713-724.

24. Clark, I. A., Cowden, W. B., Hunt, N. H., Maxwell, L. E. \& Mackie, E. J. (1984) Br. J. Haematol. 57, 479-487.

25. Burton, G. W., Joyce, A. \& Ingold, K. V. (1983) Arch. Biochem. Biophys. 221, 281-290.

26. Clark, I. A. (1982) Klin. Wochenschr. 60, 756-758.

27. Walker, R. D., Nordmeyer, J. P. \& Koenigk, E. (1974) HoppeSeyler's Z. Physiol. Chem. 355, 495-500.

28. Cohen, G. \& Hochstein, P. (1963) Biochemistry 2, 1420-1428.

29. Aebi, H., Heiniger, J. P. \& Lauber, E. (1964) Helv. Chim. Acta 47, 1428-1440.

30. Elsayed, N. M. \& Mustafa, M. G. (1982) Toxicol. Appl. Pharmacol. 66, 319-328.

31. Sevanian, A., Hacker, A. D. \& Elsayed, N. (1982) Lipids 17, 269-277.

32. Kimball, R. E., Reddy, K., Peirce, T. H., Schwartz, L. W., Mustafa, M. G. \& Cross, C. E. (1976) Am. J. Physiol. 230, 1425-1431.

33. Yamada, K. A. \& Sherman, I. W. (1979) Exp. Parasitol. 48, 61-74.

34. Halliwell, B. (1981) Bull. Eur. Physiopath. Res. 17, 21-28.

35. Freeman, R. R. (1978) Dissertation (Australian National Univ., Canberra).

36. Roth, E. F., Gilbert, H. S., Raventos-Suarez, C., Stump, D., Tanowitz, H., Rowin, K. S. \& Nagel, R. L. (1983) Blood 62, Suppl. 1, (abstr. 12). 